\title{
Molecular identification of the traditional herbal medicines, Arisaematis Rhizoma and Pinelliae Tuber, and common adulterants via universal DNA barcode sequences
}

\author{
B.C. Moon, W.J. Kim, Y. Ji, Y.M. Lee, Y.M. Kang and G. Choi \\ K-herb Research Center, Korea Institute of Oriental Medicine, \\ Daejeon, Republic of Korea \\ Corresponding author: B.C. Moon \\ E-mail: bcmoon@kiom.re.kr \\ Genet. Mol. Res. 15 (1): gmr.15017064 \\ Received August 10, 2015 \\ Accepted October 6, 2015 \\ Published February 19, 2016 \\ DOI http://dx.doi.org/10.4238/gmr.15017064
}

\begin{abstract}
Methods to identify Pinelliae Tuber and Arisaematis Rhizoma are required because of frequent reciprocal substitution between these two herbal medicines and the existence of several closely related plant materials. As a result of the morphological similarity of dried tubers, correct discrimination of authentic herbal medicines is difficult by conventional methods. Therefore, we analyzed DNA barcode sequences to identify each herbal medicine and the common adulterants at a species level. To verify the identity of these herbal medicines, we collected five authentic species (Pinellia ternata for Pinelliae Tuber, and Arisaema amurense, A. amurense var. serratum, $A$. erubescens, and $A$. heterophyllum for Arisaematis Rhizoma) and six common adulterant plant species. Maturase K (matK) and ribulose-1,5-bisphosphate carboxylase/oxygenase large subunit $(r b c L)$ genes were then amplified using universal primers. In comparative analyses of two DNA barcode sequences, we obtained 45 species-specific nucleotides sufficient to identify each species (except $A$. erubescens with matK) and 28 marker nucleotides for each species (except $P$. pedatisecta
\end{abstract}


with $r b c L)$. Sequence differences at corresponding positions of the two combined DNA barcodes provided genetic marker nucleotides that could be used to identify specimens of the correct species among the analyzed medicinal plants. Furthermore, we generated a phylogenetic tree showing nine distinct groups depending on the species. These results can be used to authenticate Pinelliae Tuber and Arisaematis Rhizoma from their adulterants and to identify each species. Thus, comparative analyses of plant DNA barcode sequences identified useful genetic markers for the authentication of Pinelliae Tuber and Arisaematis Rhizoma from several adulterant herbal materials.

Key words: DNA barcode; Maturase K (matK); Pinelliae Tuber; Ribulose-1,5-bisphosphate carboxylase/oxygenase large subunit $(r b c \mathrm{~L})$; Arisaematis Rhizoma; Molecular authentication

\section{INTRODUCTION}

Pinelliae Tuber and Arisaematis Rhizoma are well-known traditional herbal medicines widely used in East Asian countries including Korea, China, and Japan. Both of these herbal medicines are toxic perennial herbs of the monocot family, Araceae, and are mainly used as antiemetics and analgesics (Dan et al., 2004). During treatments using Asian traditional medicines, both dried tubers are used to treat dampness and transform phlegm, and can be used for phlegm-dampness in the spleen and stomach. Pinelliae Tuber specifically treats spleen and stomach phlegm-dampness while also alleviating nausea and reducing focal distention. However, Arisaematis Rhizoma, which has an acrid flavor, has a far greater dispersing action than does Pinelliae Tuber, and mainly affects liver channel phlegm-dampness. Furthermore, Arisaematis Rhizoma excels in treating wind-phlegm in the channels and collaterals, and also disperses blood and reduces swelling (Maki et al., 1987; Dan et al., 2004; Chen et al., 2012).

In the national pharmacopoeia of Korea, China, and Japan, Pinelliae Tuber is designated as the tuber of Pinellia ternata only; however, other Pinellia species, as well as some additional species, including those belonging to Typhonium and Arisaema species, are commonly misused as this herbal medicine (Korea Institute of Oriental Medicine, 2015). In particular, tubers of Pinellia tripartita, Pinellia pedatisecta, Arisaema erubescens, Arisaema yunnanense, and Typhonium flagelliforme are commonly distributed as Pinelliae Tuber in medicinal markets because of the similarity of the dried tubers of these plants to those of $P$. ternata, as well as their short growing period and high production yield compared to P. ternata (Lin et al., 2006; Cui et al., 2008; Liu and Guo, 2010). Arisaematis Rhizoma, another herbal medicine with similar morphological characteristics to those of dried Pinelliae Tuber, is described as the tuberous rhizome of only three plant species, Arisaema amurense, A. erubescens, and Arisaema heterophyllum, in the national pharmacopoeia of Korea, China, and Japan (Korea Institute of Oriental Medicine, 2015). However, young tuberous rhizomes of diverse plant species belonging to the other Arisaema species have been incorrectly used for centuries as not only Arisaematis Rhizoma but also Pinelliae Tuber (Lin et al., 2006; Liu and Guo, 2010). Previous reports have suggested that there may be seven species and five varieties of Arisaema distributed in Korea, although only two species, $A$. amurense and $A$. heterophyllum, are official medicinal ingredients (Ko and Kim, 1985; Korea Institute of Oriental Medicine, 2015). Thus, 
other Arisaema species, including A. takesimense, A. serratum, A. ringens, and $A$. thumbergii are unacceptable species for use in herbal medicine. Unfortunately, differentiating between the young tuberous rhizomes of Arisaema species and Pinelliae Tuber is difficult when using dried herbal medicines. Thus, as a result of the morphological similarities in the tubers and the lack of visual differences among processed herbal drugs, it is very difficult to determine the original species or adulterants and to distinguish between Pinelliae Tuber and Arisaematis Rhizoma. Therefore, accurate and reliable methods that do not depend on the morphology of the herbal medicine are required to differentiate between authentic and inauthentic herbal materials at a species level.

DNA barcoding has been suggested for use in phylogenetic studies, species identification of diverse flowering plants, and authentication of herbal medicines (Techen et al., 2004; Guo and Ge, 2005; Zhang et al., 2007; Hollingsworth et al., 2011). The development of polymerase chain reaction $(\mathrm{PCR})$-based methods that use a diverse range of DNA polymerases and sequencing technology has enabled the discrimination of inter- and intra-species differences by comparative sequence analysis of various DNA barcode regions in the chloroplast genome (Kress et al., 2005; Sucher and Carles, 2008). Among the diverse DNA barcode candidates, maturase K (matK) and ribulose-1,5-bisphosphate carboxylase/oxygenase large subunit $(r b c L)$ are universal plant DNA barcodes that are used by the Consortium for the Barcode of Life (CBOL, 2009). These candidates are widely used as genetic markers to identify plant species and to analyze phylogenetic relationships (Kato et al., 1998; Kress and Erickson, 2007; Saarela et al., 2013). Chloroplast matK and $r b c L$ DNA barcode regions have been used to identify species of diverse plant taxa, including herbal materials, and to clarify taxonomic origins (Cabrera et al., 2008; Sucher and Carles, 2008; Guo et al., 2011; Chao et al., 2014). In particular, DNA barcoding can provide objective tools that permit the authentication and identification of herbal medicines, especially for medicines that are often sold as dried slices or powders. However, there are no reports of molecular identification tools (and phylogenetic relationships) used to authenticate official plant species of the important traditional medicines Pinelliae Tuber and Arisaematis Rhizoma.

In this study, we analyzed the sequences of the matK and $r b c L$ universal DNA barcode regions to identify 10 plant species and one variety, which have been used as authentic herbal medicines (Pinelliae Tuber and Arisaematis Rhizoma), as well as common adulterants. Their phylogenetic relationships were also elucidated. In a comparative analysis of the two DNA barcode sequences, we obtained several marker nucleotides sufficient to authenticate each species and clarified the phylogenetic relationships of the analyzed plant species. These results provide useful information and tools that can be used to distinguish Pinelliae Tuber and Arisaematis Rhizoma and for the accurate discrimination and authentication of herbal materials and adulterants at a species level.

\section{MATERIAL AND METHODS}

\section{Plant materials}

A total of 60 genotypes from 10 species and one variety, including seven from $A$. amurense, six from $A$. amurense var. serratum, three from $A$. erubescens, six from $A$. heterophyllum, six from A. takesimense, seven from $A$. serratum, six from $A$. ringens, five from $P$. ternata, four from $P$. tripartita, six from P. pedatisecta, and four from T. flagelliforme, were used in the analysis (Table 1). Samples were collected from different native habitats in Korea and China and stored at $-70^{\circ} \mathrm{C}$ after freezing in liquid nitrogen. All plant materials were given accession numbers, and specimens were 
preserved in the Korean Herbarium of Standard Herbal Resources (KHSHR) at the Korea Institute of Oriental Medicine. Species identification was performed by the Classification and Identification Committee of the KIOM, which comprises nine experts in the fields of plant taxonomy, botany, pharmacognosy, and herbology.

\section{Preparation of genomic DNA}

Genomic DNA was extracted from fresh leaves stored at $-70^{\circ} \mathrm{C}$ and from herbal medicines using a DNeasy ${ }^{\circledR}$ Plant Mini Kit (Qiagen, Valencia, CA, USA) according to the manufacturer protocol. DNA concentrations and purities were determined by spectrophotometry (Nanodrop ND-1000, Nanodrop, Wilmington, DE, USA) and 1.5\% agarose gel electrophoresis with known standards. For PCR amplification, the final concentration of each DNA sample was approximately $20 \mathrm{ng} / \mu \mathrm{L}$ in TE buffer.

\section{Amplification of DNA barcodes}

The matK and $r b c L$ genes were amplified using matK AF (5'-CTA TAT CCA CTT ATC TTT CAG GAG T-3') and matK 8R (5'-++AAA GTT CTA GCA CAA GAA AGT CGA-3') primers for $\sim 1.3$ $\mathrm{kb}$ of the matK region, and rbcL F (5'-ATG TCA CCA CAA ACA GAA ACT AAA GC-3') and rbcL R (5'-TCC TTT TAG TAA AAG ATT GGG CGG AG-3') primers for $~ 1.5 \mathrm{~kb}$ of the rbcL region, as previously described (Olmstead and Reeves, 1995; Kato et al., 1998). PCRs were performed in $50-\mu \mathrm{L}$ reaction solutions containing $10 \mathrm{mM}$ Tris- $\mathrm{HCl}, \mathrm{pH} 9.0,2.5 \mathrm{mM} \mathrm{MgCl}, 200 \mu \mathrm{M}$ each dNTP, $10 \mathrm{mM}\left(\mathrm{NH}_{4}\right)_{2} \mathrm{SO}_{4}, 0.5 \mathrm{U}$ Taq DNA polymerase (Solgent, Daejeon, Korea), $0.6 \mu \mathrm{M}$ each primer, and 10-20 ng template DNA. DNA amplification was performed on a DNA Engine Dyad ${ }^{\circledR}$ PTC0220 (Bio-Rad, Foster City, CA, USA). The parameters used were $95^{\circ} \mathrm{C}$ for $5 \mathrm{~min}$, followed by 35 cycles of $30 \mathrm{~s}$ at $95^{\circ} \mathrm{C}, 1 \mathrm{~min}$ at $55^{\circ} \mathrm{C}$, and $2 \min$ at $72^{\circ} \mathrm{C}$, and a final extension for $10 \min$ at $72^{\circ} \mathrm{C}$. PCR products were separated on $1.5 \%$ agarose gels with a 1-kb plus DNA ladder (Solgent) and visualized with ethidium bromide staining under ultraviolet light.

\section{Analysis of nucleotide sequences for the identification of marker nucleotides and phylogenetic relationships}

The matK and rbcL DNA fragments amplified from 60 individual plant samples were retrieved from agarose gels with a Gel Extraction Kit (Solgent) and subcloned into the pGEM-T Easy vector (Promega, Madison, WI, USA). Nucleotide sequences of the inserted DNA fragments were determined from both strands via dideoxynucleotide chain termination using an automatic DNA sequence analyzer (ABI 3730, Applied Biosystems Inc., Foster City, CA, USA). The nucleotide sequences of the matK and $r b c \mathrm{~L}$ genes of each species were registered in $\mathrm{NCBI}$ GenBank (Table 1).

To identify marker nucleotides that could be used to authenticate each species using matK and $\mathrm{rbcL}$ gene sequences, the 60 sample sequences were multiple sequences aligned and edited using ClustalW implemented in biological sequence editing software (BioEdit, version 7.2.5) (Hall, 1999). The resulting species-specific indels and substitutions were selected as marker nucleotides from matK and $r b c L$, respectively, and the combination of two DNA barcode regions for the discrimination of each species. 
For analysis of sequence variability between and within species, 60 complete sample sequences of matK and $r b c L$ were compared using the DNADist DNA distance matrix in the BioEdit software. For the generation of phylogenetic trees, 60 complete matK and $r b c \mathrm{~L}$ sequences were analyzed with the DNADist neighbor-joining and unweighted pair group methods using arithmetic algorithm (UPGMA) methods in BioEdit (Hall, 1999).

\section{RESULTS}

\section{Characteristics of DNA barcode regions}

Fragments of the matK and $r b c L$ genes were successfully amplified at the expected lengths of approximately 1300 and $1500 \mathrm{bp}$, respectively, in all 60 samples. The sequences of the resulting DNA fragments were determined using the universal vector primers, T7 and SP6, after subcloning into the pGEM-Teasy system, and individual sequences were registered with GenBank (Table 1). The complete matK and rbcL genes were 1286 and 1517 bp in length in all of the analyzed samples except for T. flagelliforme in which matK was 1292 bp (Table 2). In the comparative alignment of matK sequences, all samples were aligned with a length of $1292 \mathrm{bp}$, and showed sequence variability that ranged from 0.0000-0.0047 and 0.0000-0.0393 at inter- and intra-species levels, respectively (Table 2). The $r b c L$ sequences were aligned in the same length with sequences (1517 bp) showing 0.0000-0.0046 and 0.0000-0.0194 inter- and intra-species sequence variability, respectively (Table 2 ).

\section{Identification of species-specific marker nucleotides}

To determine species-specific sequences for the identification of individual species, a comparative analysis was performed that depended on 60 complete samples from both the matK and $r b c L$ gene regions. In the sequence comparisons, nucleotide substitutions that were observed only at an inter-species level were found at 11 positions in $A$. erubescens, 21 positions in $A$. heterophyllum, 8 positions in $A$. serratum and $A$. takesimense, 10 positions in $A$. ringens, 35 positions in $P$. ternata, 38 positions in $P$. tripartita, 34 positions in $P$. pedatisecta, and 44 positions in T. flagelliforme, against $A$. amurense and its variety in the maximized alignment of each matK region (Table 2 and Figure S1). However, we did not identify any distinct sequence variability between $A$. amurense and $A$. amurense var. serratum, and between $A$. serratum and $A$. takesimense at the species level, for both of the DNA barcodes examined. These results strongly support the view that $A$. amurense var. serratum and $A$. takesimense is a taxonomic synonym of $A$. amurense and A. serratum, respectively, in The Plant List (2013). Therefore, we classified and further analyzed $A$. amurense var. serratum and $A$. takesimense as the same species as $A$. amurense and $A$. serratum, respectively.

In the maximized alignment of each $r b c L$ region, substitutions were observed in $13,12,5,8,19$,

19,18 , and 23 positions versus $A$. amurense, with respect to the same species order mentioned above for the matK region (Table 2 and Figure S2). Of these, species-specific marker nucleotides that could be used to identify each species were obtained at six positions specific to $A$. amurense and its variety, five positions for $A$. heterophyllum, one position for $A$. serratum and $A$. takesimense, two positions for $A$. ringens, three positions for $P$. ternata, five positions for $P$. tripartita, two positions for $P$. pedatisecta, and 22 positions (substitutions) and a 6-bp insertion for T. flagelliforme (Table 3 and Figure S1). 
Table 1. Summary of information regarding plant materials.

\begin{tabular}{|c|c|c|c|c|c|}
\hline \multicolumn{2}{|l|}{ Names } & \multirow{2}{*}{ Source } & \multirow{2}{*}{ Sample name } & \multicolumn{2}{|c|}{ GenBank accession No. } \\
\hline Scientific name & Herbal & & & matK & $r b c L$ \\
\hline \multirow{7}{*}{$\begin{array}{l}\text { Arisaema } \\
\text { amurense Maxim. }\end{array}$} & \multirow{22}{*}{$\begin{array}{l}\text { Arisaematis Rhizoma } \\
\text { (Tian Nan Xing, Cheon } \\
\text { Nam Seong) }\end{array}$} & Gwanavana. Jeonnam. Korea & AA KY & KT025764 & KT025714 \\
\hline & & Yanasan. Gveonanam. Korea & AA YS & KT025765 & KT025715 \\
\hline & & Jeiu. Jeiu. Korea & AA JJ & KT025766 & KT025716 \\
\hline & & Tonaveona. Gveonanam. Korea & AA TY & KT025767 & KT025717 \\
\hline & & Inie. Ganawon. Korea & AA IJ & KT025768 & KT025718 \\
\hline & & Janasu. Jeonbuk. Korea & AA JS & KT025769 & KT025719 \\
\hline & & Ansan, Gveonqai, Korea & AA AS & KT025770 & KT025720 \\
\hline \multirow{6}{*}{$\begin{array}{l}\text { Arisaema } \\
\text { amurense var. } \\
\text { serratum Nakai }\end{array}$} & & Woniu. Ganawon. Korea & AAS WJ & KT025771 & KT025721 \\
\hline & & Jeiu. Jeiu. Korea & AAS JJ & KT025772 & KT025722 \\
\hline & & Tonaveona. Gveonanam. Korea & AAS TY & KT025773 & KT024723 \\
\hline & & Yeonacheon. Gveonabuk. Korea & AAS YC & KT025774 & KT025724 \\
\hline & & Gongju, Chungnam, Korea & AAS_GJ & KT025775 & KT025725 \\
\hline & & Ansan. Gveonqai, Korea & AAS AS & KT025776 & KT025726 \\
\hline \multirow{3}{*}{$\begin{array}{l}\text { Arisaema } \\
\text { erubescens (Wall.) } \\
\text { Schott }\end{array}$} & & Guigang, Guangxi, China & AE_QS1 & KT025777 & KT025727 \\
\hline & & Guigang, Guangxi, China & AE_QS2 & KT025778 & KT025728 \\
\hline & & Guigang, Guangxi, China & AE_QS3 & KT025779 & KT025729 \\
\hline \multirow{6}{*}{$\begin{array}{l}\text { Arisaema } \\
\text { heterophyllum } \\
\text { Blume }\end{array}$} & & Sancheona. Gveonanam. Korea & $\mathrm{AH} S \mathrm{C}$ & KT025780 & KT025730 \\
\hline & & Gwanavana. Jeonnam. Korea & $\mathrm{AH} \mathrm{KY}$ & KT025781 & KT025731 \\
\hline & & Tonaveona. Gveonanam. Korea & AH TY & KT025782 & KT025732 \\
\hline & & Cheonavana. Chunanam, Korea & $\mathrm{AH} \mathrm{CY}$ & KT025783 & KT025733 \\
\hline & & Ansan, Gveonqqi, Korea & AH AS & KT025784 & KT025734 \\
\hline & & Gapveona. Gveonaai. Korea & $\mathrm{AH}$ GP & KT025785 & KT025735 \\
\hline \multirow{7}{*}{$\begin{array}{l}\text { Arisaema serratum } \\
\text { (Thunb.) Schott }\end{array}$} & \multirow{7}{*}{ a } & Gwanavana. Jeonnam. Korea & AS KY & KT025786 & KT025736 \\
\hline & & Janasu. Jeonbuk. Korea & AS JS & KT025787 & KT025737 \\
\hline & & Geoie, Gveonanam, Korea & AS GJ & KT025788 & KT025738 \\
\hline & & Jeiu. Jeiu. Korea & AS JJ & KT025789 & KT025739 \\
\hline & & Inie. Ganawon. Korea & AS IJ & KT025790 & KT025740 \\
\hline & & Ulleuna. Gveonabuk. Korea & AS UR & KT025791 & KT025741 \\
\hline & & Yanbian. Jilin. China & AS JL & KT025792 & KT025742 \\
\hline \multirow{6}{*}{$\begin{array}{l}\text { Arisaema } \\
\text { takesimense Nakai }\end{array}$} & \multirow{6}{*}{ a } & Ulleuna. Gveonabuk. Korea & AT BR1 & KT025793 & KTO25743 \\
\hline & & Ulleuna. Gveonabuk. Korea & AT BR2 & KT025794 & KT025744 \\
\hline & & Ulleuna. Gveonabuk. Korea & AT NR1 & KT025795 & KT025745 \\
\hline & & Ulleuna. Gveonabuk. Korea & AT NR2 & KT025796 & KT025746 \\
\hline & & Ulleuna. Gveonabuk. Korea & AT NSJ1 & KT025797 & KT025747 \\
\hline & & Ulleung, Gyeongbuk, Korea & AT_NSJ2 & KT025798 & KT025748 \\
\hline \multirow{6}{*}{$\begin{array}{l}\text { Arisaema ringens } \\
\text { (Thunb.) Schott }\end{array}$} & \multirow{6}{*}{$a$} & Geoie. Gveonanam. Korea & AR GJ & KT025799 & KT025749 \\
\hline & & Tonaveona, Gveonanam, Korea & AR TY & KT025800 & KT025750 \\
\hline & & Jeiu. Jeju. Korea & AR GJA & KT025801 & KT025751 \\
\hline & & Jeiu. Jeiu. Korea & AR JC & KT025802 & KT024752 \\
\hline & & Seoawipo, Jeiu. Korea & AR SGP & KT025803 & KT025753 \\
\hline & & Jeiu. Jeiu. Korea & AR CJ & KT025804 & KT025754 \\
\hline \multirow{5}{*}{$\begin{array}{l}\text { Pinellia ternata } \\
\text { (Thunb.) Makino }\end{array}$} & \multirow{5}{*}{$\begin{array}{l}\text { Pinelliae Tuber } \\
\text { (Ban xia, Ban Ha)b }\end{array}$} & Chengdu, Sichuan, China & PI1 & KT025805 & KT025695 \\
\hline & & Seogwipo, Jeiu, Korea & PT2 & KT025806 & KT025696 \\
\hline & & Sacheon. Gveonanam. Korea & PT3 & KT025807 & KT025697 \\
\hline & & Sanmenxia. Henan, China & PT4 & KT025808 & KT025698 \\
\hline & & Quivana. Guizhou. China & PT5 & KT025809 & KT025699 \\
\hline \multirow{4}{*}{$\begin{array}{l}\text { Pinellia tripartita } \\
\text { (Blume) Schott }\end{array}$} & \multirow{4}{*}{$a$} & Geoje, Gyeongnam, Korea & PTP GJ & KT025810 & KT025700 \\
\hline & & Seiona. Chunanam. Korea & PTP SJ & KT025811 & KT025701 \\
\hline & & Jeoniu. Jeonbuk. Korea & PTP JEJ & KT025812 & KT025702 \\
\hline & & Tonaveona. Greonanam. Korea & PTP TY & KT025813 & KT025703 \\
\hline
\end{tabular}

Continued on next page 
Table 1. Continued.

\begin{tabular}{|c|c|c|c|c|c|}
\hline \multicolumn{2}{|l|}{ Names } & \multirow{2}{*}{ Source } & \multirow{2}{*}{ Sample name } & \multicolumn{2}{|c|}{ GenBank accession No. } \\
\hline Scientific name & Herbal & & & matK & $r b c L$ \\
\hline \multirow{6}{*}{$\begin{array}{l}\text { Pinellia } \\
\text { pedatisecta Schott }\end{array}$} & \multirow{6}{*}{$\begin{array}{l}a(\text { Hu Zhang, Ho Jang } \\
\text { Nam Seong }) b\end{array}$} & Dinaxi. Gansu. China & $P P$ Cn GS & KT025814 & KT025704 \\
\hline & & Changsha. Hunan. China & $\mathrm{PP} \mathrm{Cn} \mathrm{HN}$ & KT025815 & KT025705 \\
\hline & & Quivana. Guizhou. China & PP Cn QY & KT025816 & KT025706 \\
\hline & & Kaili. Guizhou. China & $\mathrm{PP} \mathrm{Cn} \mathrm{KL}$ & KT025817 & КT025707 \\
\hline & & Anquo. Hebei. China & $P P C n A G$ & KT025818 & KT025708 \\
\hline & & Harbin, Heilonqiiang. China & $\mathrm{PP} \mathrm{Cn} \mathrm{HB}$ & KT025819 & KT025709 \\
\hline \multirow{4}{*}{$\begin{array}{l}\text { Typhonium } \\
\text { flagelliforme } \\
\text { (Lodd.) Blume (= } \\
\text { Arum flagelliforme } \\
\text { Lodd.) }\end{array}$} & \multirow{4}{*}{$\begin{array}{l}\text { a(Shui Ban Xia, Su } \\
\text { Ban Ha)b }\end{array}$} & Pingnan, Guangxi, China & TF_Cn_PN1 & KT025820 & KT025710 \\
\hline & & Pingnan, Guangxi, China & TF_Cn_PN2 & KT025821 & KT025711 \\
\hline & & Baoxing, Sichuan, China & TF_Cn_PN1 & KT025822 & KT025712 \\
\hline & & Baoxing, Sichuan, China & TF_Cn_PN2 & KT025824 & KT025713 \\
\hline
\end{tabular}

${ }^{a}$ There is no appropriate official herbal name. ${ }^{b} \mathrm{Common}$ inauthentic herbal names used in China and Korea, respectively.

Table 2. Analysis of the two DNA barcode regions used in this study.

\begin{tabular}{|c|c|c|c|c|c|c|}
\hline \multirow{2}{*}{ Barcode } & \multirow{2}{*}{ Species } & \multirow{2}{*}{$\begin{array}{c}\text { Constant } \\
\text { length (bp) }\end{array}$} & \multirow{2}{*}{$\begin{array}{c}\text { Aligned } \\
\text { length (bp) }\end{array}$} & \multirow{2}{*}{$\begin{array}{c}\text { Intra-species } \\
\text { variability }\end{array}$} & \multicolumn{2}{|c|}{ Inter-species variation } \\
\hline & & & & & Indels & Substitutions \\
\hline \multirow{11}{*}{ matK } & A. amurense & 1286 & 1292 & $0-0.0008$ & 0 & 0 \\
\hline & A. amurense var. serratum & 1286 & 1292 & $0-0.0016$ & 0 & 0 \\
\hline & A. erubescens & 1286 & 1292 & 0 & 0 & 11 \\
\hline & A. heterophyllum & 1286 & 1292 & 0 & 0 & 21 \\
\hline & A. serratum & 1286 & 1292 & 0 & 0 & 8 \\
\hline & A. takesimense & 1286 & 1292 & 0 & 0 & 8 \\
\hline & A. ringens & 1286 & 1292 & 0 & 0 & 10 \\
\hline & P. ternata & 1286 & 1292 & $0-0.0016$ & 0 & 35 \\
\hline & P. tripartita & 1286 & 1292 & $0-0.0008$ & 0 & 38 \\
\hline & P. pedatisecta & 1286 & 1292 & $0-0.0023$ & 0 & 34 \\
\hline & T. flagelliforme & 1292 & 1292 & $0.0015-0.0047$ & 6 & 44 \\
\hline \multirow{11}{*}{$r b c L$} & A. amurense & 1517 & 1517 & $0-0.0007$ & 0 & 0 \\
\hline & A. amurense var. serratum & 1517 & 1517 & 0 & 0 & 0 \\
\hline & A. erubescens & 1517 & 1517 & $0-0.0007$ & 0 & 13 \\
\hline & A. heterophyllum & 1517 & 1517 & 0 & 0 & 12 \\
\hline & A. serratum & 1517 & 1517 & 0 & 0 & 5 \\
\hline & A. takesimense & 1517 & 1517 & 0 & 0 & 5 \\
\hline & A. ringens & 1517 & 1517 & 0 & 0 & 8 \\
\hline & P. ternata & 1517 & 1517 & $0-0.0007$ & 0 & 19 \\
\hline & P. tripartita & 1517 & 1517 & $0.0013-0.0033$ & 0 & 19 \\
\hline & P. pedatisecta & 1517 & 1517 & 0 & 0 & 18 \\
\hline & T. flagelliforme & 1517 & 1517 & $0.0013-0.0046$ & 0 & 23 \\
\hline
\end{tabular}

Furthermore, we also obtained two marker nucleotides specific for $A$. amurense and its variety, six for $A$. erubescens, two for $A$. heterophyllum, three for $A$. serratum and $A$. takesimense, four for $A$. ringens, four for $P$. ternata, one for $P$. tripartita, and seven for $T$. flagelliforme from the 
$r b c L$ gene (Table 4 and Fiqure S2). Thus, we identified 45 nucleotide substitutions and a 6-bp insertion, which were sufficient to identify each species, excluding $A$. erubescens, using the matK gene and 28 positions, excluding P. pedatisecta, using the rbcL gene (Tables 3 and 4). These species-specific marker nucleotides from matK and $r b c \mathrm{~L}$ indicate that both of these DNA barcode regions are useful and sufficient for distinguishing between Arisaematis Rhizoma and Pinelliae Tuber as well as medicinal plant species in general.

Table 3. Summary of marker nucleotides obtained from the comparison of maturase $\mathrm{K}$ (matK) gene sequences

\begin{tabular}{|c|c|c|c|c|c|c|c|c|c|c|c|c|c|c|c|c|c|c|c|c|c|c|c|c|}
\hline Sequence position & $\omega$ & $\stackrel{\infty}{\longrightarrow}$ & 8 & $\stackrel{8}{N}$ & $\vec{\vartheta}$ & $\vec{\Xi}$ & $\stackrel{\vec{\omega}}{\omega}$ & $\vec{N}$ & $\overrightarrow{\stackrel{9}{v}}$ & 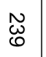 & 总 & 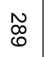 & 岕 & $\underset{\widetilde{U}}{\omega}$ & 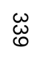 & $\stackrel{\omega}{U}$ & 岕 & 点 & to & $\stackrel{\vec{\omega}}{\circ}$ & & ज̂ & $\vec{N}$ & 忽 \\
\hline A. amurense & c & $\underline{c}$ & T & A & A & A & T & G & A & T & G & T & c & c & A & $\underline{\mathrm{c}}$ & $\underline{G}$ & & $\cdots$ & G & & c & A & A \\
\hline $\begin{array}{l}\text { A. amurense var. } \\
\text { serratum }\end{array}$ & & . & & . & & & & & & & & & & & & & & & & & & & & \\
\hline A. erubescens & r & $\mathrm{T}$ & & . & r & . & & . & - & . & & . & . & - & v & $\mathrm{T}$ & A & & $\cdots$ & , & & . & v & \\
\hline A. heterophyllum & v & $T$ & . & $\underline{G}$ & . & . & . & . & . & . & & . & . & & . & T & A & & & . & & $\underline{A}$ & & \\
\hline A. serratum & - & $\mathrm{T}$ & . & . & . & . & . & . & . & . & . & . & . & - & - & $\mathrm{T}$ & A & & -.. & - & & - & - & $\underline{\mathrm{G}}$ \\
\hline A. takesimense & r & T & . & v & v & ' & & . & . & . & , & - & v & . & & T & A & & $\cdots$ & r & & r & - & $\underline{\mathrm{G}}$ \\
\hline A. ringens & r & T & . & . & . & v & . & . & . & . & & . & . & . & . & T & A & & $\cdots$ & . & & r & v & \\
\hline P. ternata & & $\mathrm{T}$ & $\underline{\mathrm{c}}$ & . & & v & r & v & & . & & v & & $\underline{A}$ & . & $\mathrm{T}$ & A & & $\cdots$ & . & & v & $\underline{G}$ & \\
\hline P. tripartita & - & $\mathrm{T}$ & v & . & $\underline{G}$ & v & & I & . & . & & . & . & s & $\underline{G}$ & T & A & & $\cdots$ & . & & . & & \\
\hline P. pedatisecta & v & $\mathrm{T}$ & . & . & r & . & & . & - & . & & . & $\underline{A}$ & c & & $\mathrm{T}$ & A & & $\cdots$ & . & & . & & \\
\hline T. flagelliforme & I & $\mathrm{T}$ & r & - & r & I & $\underline{\mathrm{C}}$ & v & I & $\underline{\mathrm{C}}$ & $\underline{A}$ & $\underline{\mathrm{C}}$ & - & - & & T & A & & STAT & $\underline{I}$ & & & & \\
\hline Sequence position & 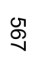 & ป్ర & $\underset{\omega}{\infty}$ & 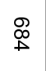 & 赵 & $\vec{v}$ & స్టు & $\mathbb{N}^{2}$ & है & ळొ & ڤ్ & $\stackrel{\infty}{\sim}$ & 意 & 尽 & 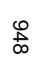 & \begin{tabular}{l}
0 \\
\hdashline \\
$\infty$
\end{tabular} & ஜ & 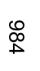 & $\stackrel{\square}{\circledR}$ & 总 & 客 & $\overrightarrow{0}$ & $\overrightarrow{\overrightarrow{\vec{v}}}$ & $\overrightarrow{\overrightarrow{\vec{A}}}$ \\
\hline A. amurense & $\mathrm{T}$ & G & c & $\mathrm{T}$ & c & $\underline{A}$ & A & A & G & c & C & A & c & $\underline{\mathrm{G}}$ & T & T & $\mathrm{T}$ & c & $\mathrm{T}$ & $\mathrm{T}$ & T & $\mathrm{T}$ & T & I \\
\hline $\begin{array}{l}\text { A. amurense var. } \\
\text { serratum }\end{array}$ & . & v & . & . & , & . & & . & , & . & & . & . & r & . & . & . & . & - & & - & . & & V \\
\hline A. erubescens & . & - & - & - & - & G & . & - & . & . & . & . & - & A & . & - & . & - & - & - & - & $\cdot$ & . & A \\
\hline A. heterophyllum & s. & - & - & - & - & G & . & - & v & $\underline{A}$ & & I & $\underline{A}$ & A & - & & r & - & - & - & - & $\cdot$ & . & A \\
\hline A. serratum & - & - & - & - & - & G & . & . & s & v & . & v & . & A & $\therefore$ & - & r & - & - & . & - & $\cdot$ & - & G \\
\hline A. takesimense & r & . & r & . & - & G & r & 冫 & r & 冫 & . & r & v & A & s & 冫 & s & . & - & v & 冫 & . & ᄂ & G \\
\hline A. ringens & - & . & - & - & - & G & - & . & - & v & - & . & - & A & $\underline{\mathrm{C}}$ & . & r & - & . & $\underline{\mathrm{C}}$ & . & . & . & A \\
\hline$P$. ternata & . & - & $\mathrm{T}$ & - & - & G & - & 冫 & - & v & r & . & $\therefore$ & c & $\therefore$ & . & r & - & - & v & . & $\cdot$ & . & G \\
\hline P. tripartita & ‘ & . & $\mathrm{T}$ & • & - & G & ' & $\therefore$ & . & v & . & 冫 & 冫 & $\mathrm{C}$ & 冫 & . & ' & $\underline{A}$ & . & 冫 & . & $\underline{\mathrm{G}}$ & . & $\mathrm{G}$ \\
\hline P. pedatisecta & v & - & $\mathrm{T}$ & - & r & G & r & . & r & v & $\underline{I}$ & ' & . & c & . & v & v & v & . & . & v & - & . & G \\
\hline T. flagelliforme & $\underline{\mathrm{G}}$ & $\underline{A}$ & $\underline{A}$ & $\underline{c}$ & I & G & $\underline{G}$ & I & A & . & & . & . & A & & $\underline{\mathrm{c}}$ & $\underline{\mathrm{c}}$ & - & $\underline{A}$ & . & $\underline{\mathrm{C}}$ & . & $\underline{\mathrm{G}}$ & G \\
\hline
\end{tabular}

Sequence positions indicate the number of aligned matK region nucleotide positions of all species. Dots represent sequence residues identical to those in Arisaema amurense. Underlined characters indicate unique species-specific marker nucleotides. 


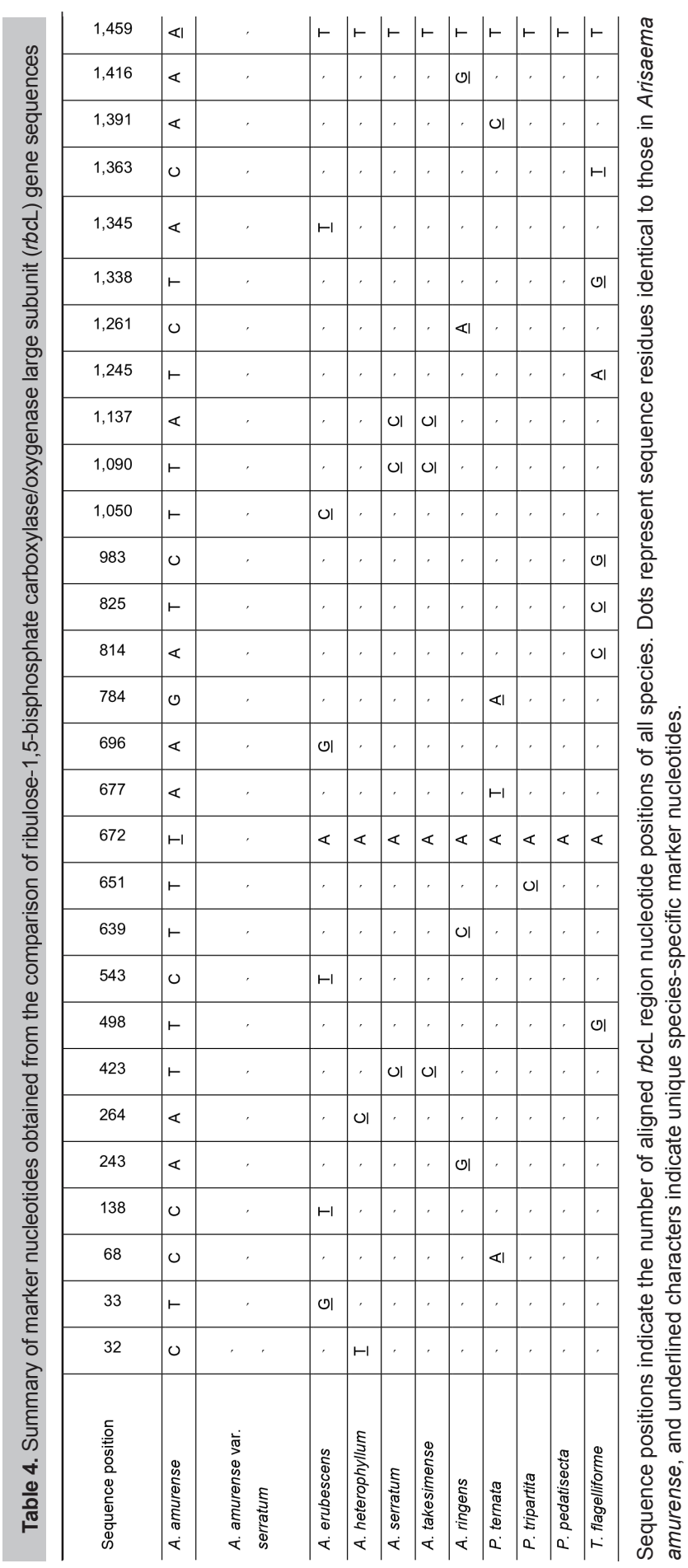




\section{Phylogenetic relationships of Arisaematis Rhizoma- and Pinelliae Tuber-related plant species}

A phylogenetic tree was constructed based on each full matK and $r b c L$ sequence (from 60 samples obtained from 10 species and one variety) using the neighbor-joining method. All samples were classified into nine distinct groups depending on the species, irrespective of the distribution areas in both matK and rbcL (Figure 1). However, $A$. amurense var. serratum and $A$. takesimense formed the same group as $A$. amurense and $A$. serratum, respectively (Figure 1 ). These results strongly support that $A$. amurense var. serratum is the same species as $A$. amurense, and that $A$. takesimense is the same species as $A$. serratum. When comparing the two herbal medicines, four species related to Pinelliae Tuber ( $P$. ternata, $P$. tripartita, $P$. pedatisecta, and $T$. flagelliforme) formed one clade, but others related to Arisaematis Rhizoma ( $A$. amurense, A. erubescens, $A$. heterophyllum, $A$. serratum, $A$. takesimense, and $A$. ringens) did not form a distinct clade for both DNA barcode regions (Figure 1). Thus, the results of phylogenetic analyses between the official and inauthentic species of Arisaematis Rhizoma did not correlate with the taxonomic positions and medicinal applications noted in the pharmacopoeia. Furthermore, the results did not provide criteria for distinguishing between the two herbal medicines, Arisaematis Rhizoma and Pinelliae Tuber.

\section{Identification of authentic Arisaematis Rhizoma and Pinelliae Tuber}

To distinguish authentic herbal medicines on the basis of DNA barcodes, an ideal DNA barcode should exhibit diverse marker nucleotides and an ability to clearly identify species. It should be sufficient to identify authentic herbal medicines by comparing individual DNA barcodes because diverse nucleotide substitutions can be observed between authentic and inauthentic species in both DNA barcodes even though the two plant species, $A$. erubescens and $P$. pedatisecta, did not reveal distinct species-specific marker nucleotides in matK and $r b c \mathrm{~L}$, respectively (Tables 3 and 4). For the authentication of Arisaematis Rhizoma from inauthentic plant materials and other closely related species such as Pinellia and Typhonium, we suggest that a comparison of both matK and rbcL DNA barcode sequences should be used, because $A$. erubescens could not be clearly differentiated from other species in the matK analysis (Table 3). We also recommend a comparison of both DNA barcode sequences to discriminate authentic Pinelliae Tuber from other adulterant plant materials for similar reasons to those stated above, but with $P$. pedatisecta in the rbcL analysis (Table 4). In addition to marker nucleotides, the phylogenetic analysis of individual DNA barcode sequences clearly classified the two herbal medicines according to the species examined. Therefore, phylogenetic analysis using DNA barcodes provided useful information to discriminate authentic herbal medicines at a species level. These combined results obtained from DNA barcode marker nucleotides of both matK and rbcL gene sequences, and the phylogenetic relationships among the species, can be used to distinguish between Arisaematis Rhizoma and Pinelliae Tuber and between the other analyzed species. 
A

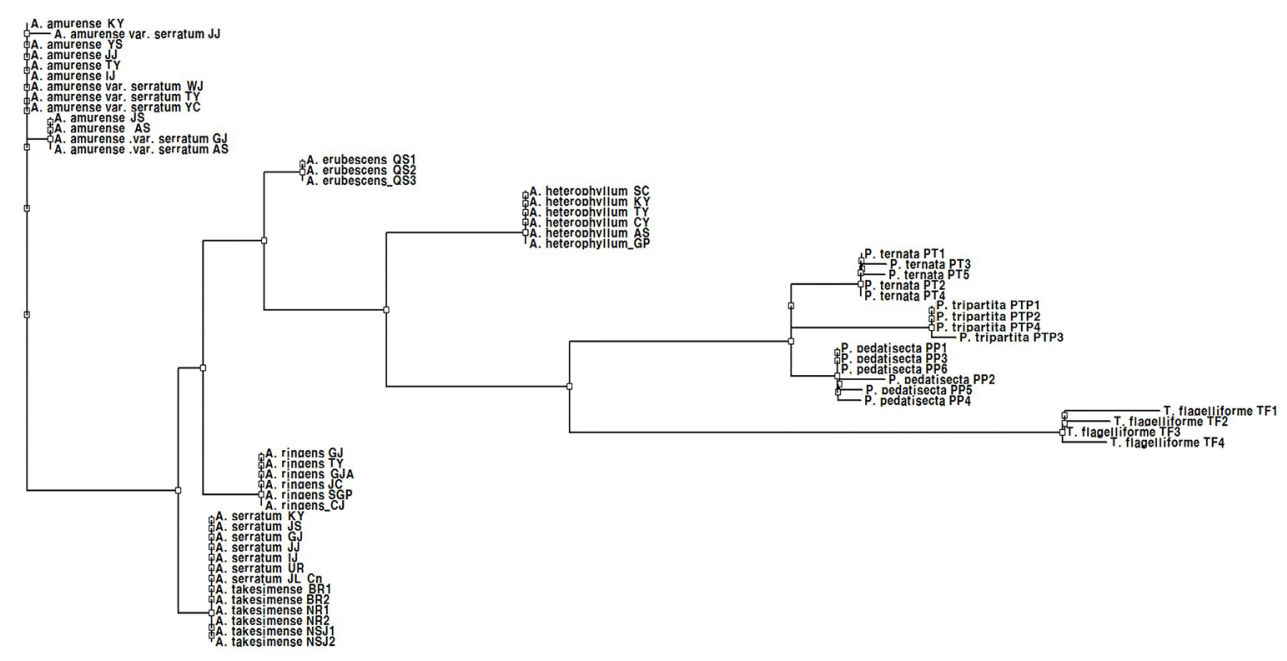

$\underline{0.001}$

B

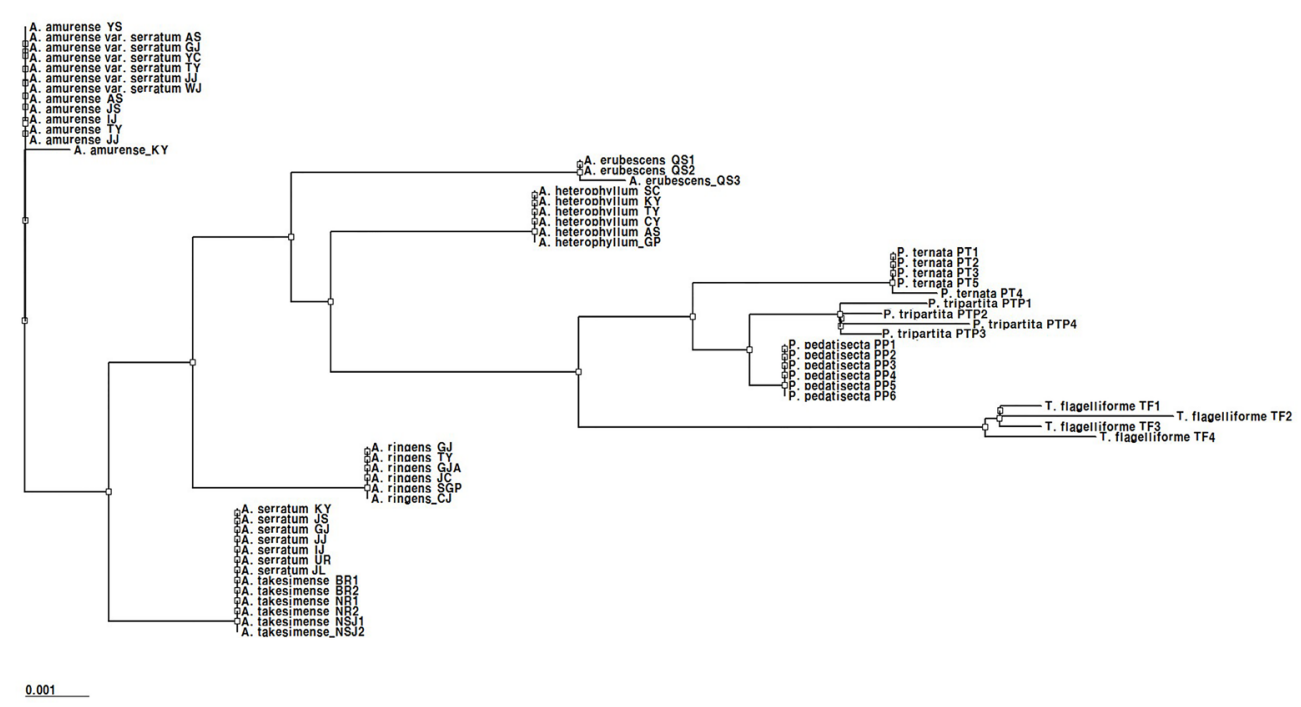

Figure 1. Phylogenetic tree of official Arisaematis Rhizoma and Pinelliae Tuber medicinal plant species and closely related plant species constructed on the basis of matK and rbcL DNA barcode sequences. A. Phylogenetic tree of matK barcode sequences. B. Phylogenetic tree of rbcL barcode sequences.

\section{DISCUSSION}

Accurate authentication of species is important for standardization and quality control of traditional herbal medicines (Sucher and Carles, 2008). Since processed herbal materials, such as dried slices or powders, are very difficult to accurately identify, we performed analyses to 
distinguish between Arisaematis Rhizoma and Pinelliae Tuber, and also to authenticate the official species among ten species and one variety. We incorporated original plant species described in the Korean pharmacopoeia, which included common adulterants of Arisaematis Rhizoma and Pinelliae Tuber (Table 1). Thus, we included $A$. serratum, $A$. takesimense, and $A$. ringens for the typical adulterants of Arisaematis Rhizoma, and P. tripartita, $P$. pedatisecta, and T. flagelliforme for the typical adulterants of Pinelliae Tuber, with the official plant species $A$. amurense, $A$. erubescens, $A$. heterophyllum, and $P$. ternata in our analyses. In addition, we examined all species related to both herbal medicines for clear authentication of these medicinal plants at a species level. Therefore, this study was designed to clarify the differences between Arisaematis Rhizoma and Pinelliae Tuber and to authenticate plant species closely related to these herbal medicines, including common adulterants, on the basis of distinct genetic information for each species.

To provide genetic tools and information for authenticating official herbal medicines, we performed a comparison of DNA barcode sequences using related plant materials. A desirable DNA barcode should contain sufficient sequence variation to permit species discrimination and show significant inter-species differences with minimal intra-species variation, together with convenient flanking regions for PCR amplification and sequencing (CBOL Plant Working Group, 2009; Guo et al., 2011). The CBOL proposed using a combination of matK and $r b c L$ as a plant barcode due to the above-mentioned features (Saarela et al, 2013). The two universal DNA barcodes used in this study, matK and $r b c L$, were successfully amplified by the universal primer and were found to exhibit interspecies specific variations that could be used to identify each species with little intra-species sequence divergence (Figures S1 and $\underline{\mathbf{S} 2}$ ). Therefore, we further analyzed these two regions as potential barcodes for authenticating official Arisaematis Rhizoma and Pinelliae Tuber from adulterant materials.

When comparing individual DNA barcode sequences, matK and rbcL showed high sequence divergence, and both regions could be employed as unique barcodes for the authentication of Arisaematis Rhizoma and Pinelliae Tuber (Tables 3 and 4). Although individual DNA barcodes were sufficient to discriminate each species, we strongly recommend using both barcode regions, as suggested for discriminating Scutellaria baicalensis from its adulterants in a previous report, because matK and $r b c \mathrm{~L}$ did not exhibit unique marker nucleotides for $A$. erubescens and $P$. pedatisecta, respectively, and multilocus DNA barcodes are more effective for identification of plant species (Stoeckle, 2003; Guo et al., 2011; Purushothaman et al., 2014). In addition to the use of both matK and $r b c L$ barcode regions, we also suggest using phylogenetic analyses to authenticate herbal medicines and their adulterants because phylogenetic clustering provides additional information that can be used for species discrimination on the basis of sequence divergence and co-relationships.

The genus Arisaema, which is a member of the Araceae family, contains more than 130 species worldwide, and 660 plant names along with various synonyms, are recorded in the Plant List (Gusman and Gusman 2002; The Plant List 2013). A. amurense var. serratum and $A$. takesimense are suggested as synonyms of $A$. amurense and $A$. serratum, respectively, although these two species are separately classified independent plant taxa in Korea (Ko and Kim, 1985; Kang et al., 2003; The Plant List, 2013). However, both the matK and $r b c L$ barcode sequences of $A$. amurense var. serratum and $A$. takesimense were identical to those of $A$. amurense and A. serratum (Tables 3 and 4 , Figures S1 and $\underline{\mathbf{S 2}}$ ). In this study, therefore, we established genetic tools that can be used for the authentication of two official herbal medicines, Arisaematis Rhizoma and Pinelliae Tuber. Furthermore, we provided objective evidence for the taxonomic classification of Korean Arisaema species via species-specific marker nucleotides and phylogenetic analyses. 


\section{Conflicts of interest}

The authors declare no conflict of interest.

\section{ACKNOWLEDGMENTS}

We thank the Classification and Identification Committee of the KIOM for the critical identification of plant materials and for helpful discussions. Research mainly supported by the Development of Basic Technologies for the Domestic Cultivation of Herbal Medicine Resources (\#K15421) and partially supported by the Construction of the Basis for Practical Application of Herbal Resources (\#K12020) funded by the Ministry of Science, ICT, and Future Planning (MSIP) of Korea to the Korea Institute of Oriental Medicine.

\section{REFERENCES}

Cabrera LI, Salazar GA, Chase MW, Mayo SJ, et al. (2008). Phylogenetic relationships of aroids and duckweeds (Araceae) inferred from coding and noncoding plastid DNA. Am. J. Bot. 95: 1153-1165. http://dx.doi.org/10.3732/ajb.0800073

CBOL Plant Working Group (2009). A DNA barcode for land plants. Proc. Natl. Acad. Sci. USA 106: 12794-12797. http://dx.doi. org/10.1073/pnas.0905845106

Chao Z, Zeng W, Liao J, Liu L, et al. (2014). DNA barcoding Chinese medicinal Bupleurum. Phytomedicine 21: 1767-1773. http://dx.doi.org/10.1016/j.phymed.2014.09.001

Chen G, Xu J, Miao X, Huan Y, et al. (2012). Characterization and antitumor activities of the water-soluble polysaccharide from Rhizoma Arisaematis. Carbohydr. Polym. 90: 67-72. http://dx.doi.org/10.1016/j.carbpol.2012.04.063

Cui GH, Tang XJ, Huang LQ and Qian ZZ (2008). [Authentication of Pinellia pedatisecta and their adulterants by AS-PCR]. Zhongguo Zhong Yao Za Zhi 33: 1109-1111.

Dan B, Steven C, Erich S and Andrew G (2004). Chinese Herbal Medicine Matria Medica. Herbs that transform phlegm and stop coughing. 3rd edn. Eastland Press. Washington, 413-421.

Guo X, Wang X, Su W, Zhang G, et al. (2011). DNA barcodes for discriminating the medicinal plant Scutellaria baicalensis (Lamiaceae) and its adulterants. Biol. Pharm. Bull. 34: 1198-1203. http://dx.doi.org/10.1248/bpb.34.1198

Guo YL and Ge S (2005). Molecular phylogeny of Oryzeae (Poaceae) based on DNA sequences from chloroplast, mitochondrial, and nuclear genomes. Am. J. Bot. 92: 1548-1558. http://dx.doi.org/10.3732/ajb.92.9.1548

Gusman $G$ and Gusman L (2002). The genus Arisaema: A monograph for botanists and nature lovers. A.R.F. Gantner Verlag KG, Lichtenstein.

Hall TA (1999). BioEdit: a user-friendly biological sequence alignment editor and analysis program for Windows 95/98/NT. Nucleic Acids Symp. Ser. 41: 95-98.

Hollingsworth PM, Graham SW and Little DP (2011). Choosing and using a plant DNA barcode. PLoS One 6: e19254. http:// dx.doi.org/10.1371/journal.pone.0019254

Kang JH, Yun JB and Ju YS (2003). The external and internal morphological standard of original plants and herbal states in 2 kinds of Arisaematis Rhizoma. Korean J. Orient. Med. Prescr 11: 213-232.

Kato H, Oginuma K, Gu Z, Hammel B, et al. (1998). Phylogenetic relationships of Betulaceae based on matK sequences with particular reference on the positions of Ostryopsis. Acta Phytotax. Geobot 49: 89-97.

Ko SC and Kim YS (1985). A taxonomic study on genus Arisaema in Korea. Kor. J. Plant Tax 15: 67-109.

Korea Institute of Oriental Medicine (2015). Defining Dictionary for Medicinal Herbs. http://boncho.kiom.re.kr/codex/. Accessed May. 25, 2015. Article in Korean.

Kress WJ and Erickson DL (2007). A two-locus global DNA barcode for land plants: the coding $r b c L$ gene complements the non-coding trnH-psbA spacer region. PLoS One 2: e508. http://dx.doi.org/10.1371/journal.pone.0000508

Kress WJ, Wurdack KJ, Zimmer EA, Weigt LA, et al. (2005). Use of DNA barcodes to identify flowering plants. Proc. Natl. Acad. Sci. USA 102: 8369-8374. http://dx.doi.org/10.1073/pnas.0503123102

Lin J, Zhou X, Gao S, Wu W, et al. (2006). Authentication of Pinellia ternata and its adulterants based on PCR with specific primers. Planta Med. 72: 844-847. http://dx.doi.org/10.1055/s-2006-931605

Liu T and Guo X (2010). Detection of Arisaema yunnanense as adulterant of traditional medicine Pinellia ternata using allelespecific diagnostic polymerase chain reaction (PCR). Afr. J. Biotechnol. 9: 8597-8601. 
Maki T, Takahashi K and Shibata S (1987). An anti-emetic principle of Pinellia ternata tuber. Planta Med. 53: 410-414. http:// dx.doi.org/10.1055/s-2006-962759

Olmstead RG and Reeves PA (1995). Evidence for the polyphyly of the Scropulariaceae based on choloroplast $r b c L$ and $n d h F$ sequences. Ann. Mo. Bot. Gard. 82: 176-193. http://dx.doi.org/10.2307/2399876

Purushothaman N, Newmaster SG, Ragupathy S, Stalin N, et al. (2014). A tiered barcode authentication tool to differentiate medicinal Cassia species in India. Genet. Mol. Res. 13: 2959-2968. http://dx.doi.org/10.4238/2014.April.16.4

Saarela JM, Sokoloff PC, Gillespie LJ, Consaul LL, et al. (2013). DNA barcoding the Canadian Arctic flora: core plastid barcodes (rbcL + matK) for 490 vascular plant species. PLoS One 8: e77982. http://dx.doi.org/10.1371/journal.pone.0077982

Stoeckle M (2003). Taxonomy, DNA and the barcode of life. BioSci 53: 796-797. http://dx.doi.org/10.1641/00063568(2003)053[0796:TDATBC]2.0.CO;2

Sucher NJ and Carles MC (2008). Genome-based approaches to the authentication of medicinal plants. Planta Med. 74: 603623. http://dx.doi.org/10.1055/s-2008-1074517

Techen N, Crockett SL, Khan IA and Scheffler BE (2004). Authentication of medicinal plants using molecular biology techniques to compliment conventional methods. Curr. Med. Chem. 11: 1391-1401. http://dx.doi.org/10.2174/0929867043365206

The Plant List (2013). Version 1.1. Published on the internet; http://www.theplantlist.org. Accessed May 25, 2015.

Zhang YB, Shaw PC, Sze CW, Wang ZT, et al. (2007). Molecular authentication of Chinese herbal materials. J. Food Drug Anal. 15: 1-9.

\section{Supplementary material}

Figure S1. Comparison of matK barcode sequences of the official Arisaematis Rhizoma and Pinelliae Tuber medicinal plants and closely related plant species. Dots $(\cdot)$ indicate identical sequences to those in $A$. amurense $\mathrm{KY}$, and dashes (-) represent gaps introduced to maximize alignment.

Figure S2. Comparison of $r b c \mathrm{~L}$ barcode sequences of official Arisaematis Rhizoma and Pinelliae Tuber medicinal plants and closely related plant species. Dots (·) indicate identical sequences to those in $A$. amurense YS.

http://www.geneticsmr.com/year2016/vol15-1/pdf/gmr7064 supplementary.pdf 